Zur Rolle der Designforschung im Kontext der Circular Economy

\section{Wettbewerbsvorteile durch nachhaltige Produkte}

\author{
Während im wissenschaftlichen Diskurs die Prinzipien der \\ Circular Economy weite Zustimmung finden, steckt die Umset- \\ zung in Unternehmen noch in den Kinderschuhen. Wie kann \\ diese Lücke geschlossen werden? Und welche Rolle kommt da- \\ bei der angewandten Designforschung zu? \\ Von Carsten Buck, Magnus Fischer und Marina Beermann
}

D as in einer ganzheitlichen Betrachtung eines Produktlebenswegs ein wesentlicher Schlüssel zur Lösung nachhaltigkeitsbezogener Herausforderungen liegt, hat der Ansatz der Circular Economy bereits in den 1990er Jahren gezeigt (vgl. Pearce/Turner 1990). Mit dem von McDonough/Braungart (2002) entwickelten Konzept des "Cradle-to-Cradle“ gelangte die Idee geschlossener (Stoff-)Kreisläufe und einer abfallfreien Wirtschaft schließlich nicht nur in wissenschaftsnahe Köpfe, sondern auch in das ein oder andere Unternehmen. Trotz vereinzelter Positivbeispiele nutzen bisher nur eine kleine Minderheit von Unternehmen die Chancen und Differenzierungspotenziale, die mit einer nachhaltigen Konzipierung ihrer Produkte entlang aller Lebenszyklusphasen (inkl. Aftersales, Use, Disposal) einhergehen würden.

\section{Neuer Designprozess}

Aufgrund stetig neuer Anforderungen (z. B. ElektroG-Novelle) wäre es für die betroffenen Unternehmen erstens ratsam, sich mit den ökonomischen sowie organisatorischen Konsequenzen auseinanderzusetzen und operative Konzepte zu entwickeln. Und zweitens zu prüfen, ob systemische Lösungen im Sinne der Circular Economy Wettbewerbsvorteile generieren könnten. Dazu gehören die Entwicklung neuer Geschäftsmodelle und Kontaktpunkte zwischen Kunden und Marke sowie die Etablierung neuer Designparameter durch ein geändertes Stoffstrommanagement.
Um Circular-Economy-fähige Produkte mit und im Unternehmen entwickeln zu können, bedarf es einer neuen Rollenzuweisung des Designprozesses. Um die hier inhärenten Ansatzpunkte nutzen zu können, wurde eine Methodik entwickelt (U. P. Analysis - Untapped Potential Analysis), mit der

I die Perspektive des Designs sowie die Prinzipien der Circular Economy (vgl. hierzu Ellen MacArthur Foundation) von Beginn an integriert werden,

I in einem partizipativen Prozess alle wesentlichen Akteure und Kompetenzen im Unternehmen einbezogen werden,

I mittels einer sukzessiven Analyse von sechs Analysefeldern ungenutzte Potenziale identifiziert werden, und

I unternehmerische Pfadabhängigkeiten und Silo-Mentalitäten aufgebrochen werden.

Im Rahmen eines Workshop-basierten Verfahrens werden die sechs Analysefelder anhand einer Checkliste mit den verschiedenen Akteuren des Unternehmens analysiert und bewertet.

- Externe und interne Risikofaktoren: Welche Risiken kommen auf das Unternehmen aufgrund von gesetzlichen und wettbewerbsbedingten Veränderungen zu?

I Originäre Produktzuschreibungen: Welche Funktion und Leistung erfüllt das Produkt primär für den Verwender?

I Produktspezifischer emotionaler Mehrwert: Welche Funktionen erfüllt das Produkt neben einem rein tech- nisch-funktionalen Nutzen für den Verwender (bspw. Symbolik, Ästhetik)?

I Möglichkeiten einer Rückgewinnung von Material und Ressourcen: Existieren bereits Strukturen zur Rückgewinnung der eingesetzten Ressourcen? Kommuniziert das Produkt seine Rückführbarkeit?

I Infrastruktur für Servicemodelle und Rücknahmesysteme: Wie ist die Verlängerung der Wertschöpfungskette hin zum Verwender technisch umsetzbar?

- Schnittpunkte zwischen Kunden und Marke: Welche zusätzlichen Kontaktpunkte lassen sich etablieren? Lassen sich neue Service- und Geschäftsmodelle implementieren?

Mithilfe der U. P. Analysis können unerschlossene Differenzierungs- und Kundenbindungspotenziale identifiziert sowie unternehmerische Nutzenpotenziale von Circular-Economy-fähigen Produkten aufgezeigt werden. Zugleich wird durch die entwickelte Methodik der angewandten Designforschung eine neue Rolle für die Entwicklung nachhaltigkeitsbezogener Lösungen zugewiesen. Eine Rolle, die aus Sicht der Autoren für die Entwicklung Circular-Economy-fähiger Produkte notwendig ist.

\section{Literatur}

Ellen MacArthur Foundation, Internet: www.ellenmacarthurfoundation.org

McDonough/W., Braungart, M. (2002): Cradle to Cradle. Remaking the way we make things. New York, North Point Print.

Pearce, D./Turner, R. K. (1990): Economics of natural resources and the environment. London, Harvester Wheatsheaf.

AUTOREN + KONTAKT

Carsten Buck ist Inhaber und Geschäfts führer, Magnus Fischer ist Mitarbeiter der Design \& Beratungsagentur ,Mutter

Mutter GmbH, Gurlittstrasse 40, 20099 Hamburg. E-Mail: buck@mutter.de, fischer@mutter.de

Dr. Marina Beermann ist seit 2013 als Strategieberaterin bei Systain Consulting $\mathrm{GmbH}$ tätig.

Dr. Marina Beermann, Systain Consulting $\mathrm{GmbH}$, Spaldingstrasse 218, 20097 Hamburg. E-Mail: marina.beermann@systain.com 